\title{
Automatic Offline Formulation of Robust Model Predictive Control Based on Linear Matrix Inequalities Method
}

\author{
Longge Zhang ${ }^{1,2}$ \\ ${ }^{1}$ Department of Mathematics and Physics, North China Electric Power University, Baoding 071003, China \\ ${ }^{2}$ State Key Laboratory of Alternate Electrical Power System with Renewable Energy Sources, North China Electric Power University, \\ Beijing 102206, China
}

Correspondence should be addressed to Longge Zhang; longgexd@163.com

Received 9 January 2013; Revised 29 March 2013; Accepted 29 March 2013

Academic Editor: Josef Diblík

Copyright (C) 2013 Longge Zhang. This is an open access article distributed under the Creative Commons Attribution License, which permits unrestricted use, distribution, and reproduction in any medium, provided the original work is properly cited.

\begin{abstract}
Two automatic robust model predictive control strategies are presented for uncertain polytopic linear plants with input and output constraints. A sequence of nested geometric proportion asymptotically stable ellipsoids and controllers is constructed offline first. Then the feedback controllers are automatically selected with the receding horizon online in the first strategy. Finally, a modified automatic offline robust MPC approach is constructed to improve the closed system's performance. The new proposed strategies not only reduce the conservatism but also decrease the online computation. Numerical examples are given to illustrate their effectiveness.
\end{abstract}

\section{Introduction}

Over the last several decades, model predictive control (MPC), for its ability to handle multivariable systems and constraints, has attracted notable attention in chemicals, food, automotive, aerospace applications, and other fields $[1,2]$. MPC consists of a step-by-step optimization technique. It calculates more than one step control signal, but only the first one is implemented, and it repeats these calculations with the new measurements at the next sampling time. Since models used in MPC are only an approximation of the real process, it is extremely important for MPC to be robust to model uncertainty, and for this reason robust MPC is still under investigation $[3,4]$. In this issue, most published research findings are based on the min-max approach, where the performance index to be minimized is computed over the worst case. However, the prohibitive online computation burden limits its application to relatively slow dynamics and small-scale processes. For this reason, the research on reducing the online computation is still a hot topic.

Generally, there are three kinds of strategies to reduce the online computation. One is based on the subdividing of the characteristic regions [5-7]. The second is to design the invariant ellipsoids offline $[8,9]$, and the third is reducing the free variables in the optimization progress [10-13]. In the first method, the parameter space is systematically subdivided into characteristic regions, piecewise-affine feedback laws are computed offline, and a robust MPC is obtained using multiparametric quadratic programming [5-7]. In the second strategy, a sequence of explicit control laws corresponding to a sequence of asymptotically stable invariant ellipsoids is constructed offline [8]. It only performs the bisection search to find the smallest ellipsoid which contains the current state and applies the designed controller online. Based on the same philosophy, this offline approach is directly extended to the nominal system [9]. The closed-loop system's feasibility and optimality are improved. Both of the offline methods in $[8,9]$ are only for uncertain polytopic linear plants in the absence of external disturbance. A family of ellipsoidal sets is precomputed offline by exploiting the viability arguments for uncertain polytopic linear plants subject to persistent disturbance. The ellipsoidal sets are designed from the inner to outer layer, and the proposed procedure is robust in several senses and numerically affordable. The above two methods share the common feature of removing the online computation to offline. In the last method, an efficient robust predictive control (ERPC) is proposed [10] using the degrees of freedom on the control horizon to find large invariant 
ellipsoids for an augmented system offline, which leaves only a simple optimization problem to be solved online. An improved method for synthesizing the constrained robust model predictive controller is proposed in [12-14]. They not only dramatically reduce the online computation burden, but also guarantee the control performance by reserving at least one free control move in the whole process. However, the conservative results are usually obtained, due to the fact that almost all the existing methods are based on the known state, and most of system's states are unknown or only partly known in practice. This can hamper the application of robust MPC.

In order to reduce the conservativeness, the output feedback control has been widely investigated. The state feedback robust MPC is combined with a state observer in [15], and the controller gain is obtained by a bisection search in a lookup table online. The system with saturated at transient or steady state is investigated in [16], and an MPC robust stability algorithm is proposed. The synthesis approach for output robust MPC with input/state constraints is proposed followed by the study of the quasi-linear parameter varying system $[17,18]$. The output feedback controllers can reduce the system's conservatism. However, they may increase the online computational burden. So it is important to design a robust MPC method with low computational burden only based on the known initial state.

In this paper, we propose an offline MPC strategy for uncertain polytopic linear plants in the presence of input and output constraints. A nest of geometric proportion asymptotically stable ellipsoids is constructed which can assure the fixed convergence speed. The nested ellipsoids share the same character if the state is in an ellipsoid at the current; it must be shrinking into the inner ellipsoid with the designed controller. Moreover, an automatic offline formulation of robust constrained MPC is proposed, which not only almost reduces the online computation to zero, but is also only based on the known initial states at the expense of a possible reduction of the performance or the memory space requirements for storing the exact regions. Finally, the modified automatic offline robust MPC is proposed to improve the performance.

The rest of this paper is organized as follows. Section 2 gives the problem description and early results. In Section 3 , the main results of this contribution are proposed. To illustrate the performance of the proposed controllers, simulation examples are presented in Section 4, and some conclusions are drawn in Section 5.

Notation. The notation used is fairly standard. $R^{n}$ is the $n$ dimensional space of real valued vectors. For a matrix $Q$ and a vector $x \in R^{n},\|x\|_{Q}^{2}=x^{T} Q x$. The matrix inequality $A>B(A \geq B)$ means that $A$ and $B$ are square symmetric matrices and $A-B$ is (semi)positive definite. $x(k)($ or $x(k \mid k))$ denotes the measured or actual value of variable $x$ at real time $k, k \in\{0,1,2, \ldots\} ; x(k+i \mid k), i \in\{0,1,2, \ldots\}$, is the predicted value of $x$ at a future prediction time $k+i$ predicted at real time $k . I$ is the identity matrix with proper dimension. The symbol $*$ denotes the corresponding transpose of the lower block part of symmetric matrices. For a real number $a,[a]$ is the biggest integer not more than $a .\left\{z_{i}\right\}$ is the sequence with nonnegative integer $i$.

\section{Problem Description and Early Results}

Consider the following time-varying uncertain system [4]

$$
\begin{gathered}
x(k+1)=A(k) x(k)+B(k) u(k), \quad k \geq 0 \\
y(k)=C x(k),
\end{gathered}
$$

where $x \in R^{n}$ is the state of the plant, $u \in R^{m}$ is the control input, and $y \in R^{l}$ is the plant output. Assume $[A(k), B(k)] \epsilon$ $\Omega=\operatorname{Co}\left\{\left[A_{1}, B_{1}\right],\left[A_{2}, B_{2}\right], \ldots,\left[A_{L}, B_{L}\right]\right\}$ for every $k \geq 0$; that is, there exist $L$ nonnegative coefficients $w_{j}(k), \sum_{j=1}^{L} w_{l}(k)=$ 1 , such that $[A(k), B(k)]=\sum_{j=1}^{L} w_{j}(k)\left[A_{j}, B_{j}\right]$. The constraints are

$$
\begin{array}{ll}
\left|u_{r}(k+i \mid k)\right| \leq u_{r, \max }, & i \geq 0, r=1,2, \ldots, m \\
\left|y_{r}(k+i \mid k)\right| \leq y_{r, \max }, & i \geq 1, r=1,2, \ldots, l .
\end{array}
$$

The purpose is to design a controller that drives the system (1) with constraints (2) to the equilibrant state and minimize the worst case infinite horizon quadratic objective function:

$$
\begin{gathered}
\min _{u(k+i \mid k)=F(k) x(k+i \mid k)} \max _{[A(k+i), B(k+i)] \in \Omega, i \geq 0} J_{\infty}(k) \\
J_{\infty}(k)=\sum_{i=0}^{\infty}\left[x(k+i \mid k)^{T} Đ x(k+i \mid k)\right. \\
\left.+u(k+i \mid k)^{T} R u(k+i \mid k)\right]
\end{gathered}
$$

with $Ð>0, R>0$. The following conditions are satisfied:

$$
\begin{array}{r}
x(k+i+1 \mid k)=A(k+i) x(k+i \mid k) \\
+B(k+i) u(k+i \mid k), \\
x(k \mid k)=x(k), \quad \forall i \geq 0 \\
\|x(k+i+1 \mid k)\|_{P(k)}^{2}-\|x(k+i \mid k)\|_{P(k)}^{2} \\
\leq-\|x(k+i \mid l)\|_{D}^{2}-\|u(k+i \mid k)\|_{R}^{2} \\
\forall[A(k+i), B(k+i)] \in \Omega, \\
i \geq 0, P(k)>0,
\end{array}
$$

where $F(k)$ is the state feedback gain. Condition (6) should guarantee cost monotone decreasing and the system's robust stability. Summing (6) from $i=0$ to $i=\infty$ the result of $\max _{[A(k+i), B(k+i)] \in \Omega, i \geq 0} J_{\infty}(k) \leq\|x(k)\|_{P(k)}^{2} \leq \gamma$ can be obtained. With the definitions $Q=\gamma P(k)^{-1}$ and $F(k)=Y Q^{-1},(6)$ and $\|x(k)\|_{P(k)}^{2} \leq \gamma$ are equivalent to the following LMIs:

$$
\begin{gathered}
{\left[\begin{array}{cccc}
Q & * & * & * \\
A_{j} Q+B_{j} Y & Q & * & * \\
Ð^{1 / 2} Q & 0 & \gamma I & * \\
R^{1 / 2} Y & 0 & 0 & \gamma I
\end{array}\right] \geq 0, \quad j \in\{1, \ldots, L\}} \\
{\left[\begin{array}{cc}
1 & * \\
x(k) & Q
\end{array}\right] \geq 0, \quad Q>0 .}
\end{gathered}
$$


The input and output constraints are satisfied if there exists symmetric matrix $X$ and $Y$ such that

$$
\begin{gathered}
{\left[\begin{array}{cc}
X & * \\
Y^{T} & Q
\end{array}\right] \geq 0 \text { with } X_{r r} \leq u_{r, \max }^{2}, \quad r=1,2, \ldots, m,} \\
{\left[\begin{array}{cr}
Z & * \\
\left(A_{j} Q+B_{j} Y\right)^{T} C^{T} & Q
\end{array}\right] \geq 0, \quad j=1, \ldots, L} \\
\text { with } Z_{r r} \leq y_{r, \max }^{2}, \quad r=1,2, \ldots, l .
\end{gathered}
$$

Thus, the problem can be solved by

$$
\min _{\gamma, Q, X, Y, Z} \gamma
$$$$
\text { s.t. (7)-(8) . }
$$

The aforementioned method is computationally prohibitive for fast and/or high-dimensional dynamic systems. Wan and Kothare [8] proposed the offline robust MPC strategy to reduce the online computation. They defined the asymptotically stable invariant ellipsoid and designed a sequence of invariant ellipsoids one inside another. A set of stabilizing state feedback laws corresponding to the ellipsoids is computed offline, so no optimization is involved online. The online computation is only the selecting of the proper feedback law using bisection search. This method supposes the state to be known, but in practice the state is usually unknown or partly known. So we propose our automatic offline MPC which only supposes the initial state is known.

\section{Automatic Offline Robust MPC}

In this section, we define the geometric proportion asymptotically stable ellipsoid and present an automatic offline approach based on it. The proposed method not only reduces the online computation dramatically, but also decreases the conservativeness.

Definition 1. Given a discrete dynamical system $x(k+1)=$ $f(x(k))$, a subset $\mathscr{E}=\left\{x \in R^{n} \mid x^{T} Q^{-1} x \leq 1\right\}$ of the space $R^{n}$ is said to be a geometric proportion asymptotically stable ellipsoid (GPASE), if it has the property that, whenever $x(k) \in$ $\mathscr{E}$, then $x(k+1) \in \mathscr{E}_{\rho}$, where $\mathscr{E}_{\rho}=\left\{x \in R^{n} \mid x^{T} Q^{-1} x \leq\right.$ $\left.\rho^{2}\right\}, 0<\rho<1$.

Remark 2. If the state of a system is in GPASE, the slowest convergence speed is more than $\rho$.

Remark 3. The condition of system's state in GPASE increases the conservatism, but it can be decreased through selecting a bigger $\rho$ near to 1 .
The speed of the closed-loop convergence can be influenced by specifying a minimum decay rate on the state $x\left(\|x(k)\| \leq \rho^{k}\|x(0)\|, 0<\rho<1\right)$ as follows:

$$
\begin{aligned}
& x(k+i+1 \mid k)^{T} Q^{-1} x(k+i+1 \mid k) \\
& \quad \leq \rho^{2} x(k+i \mid k)^{T} Q^{-1} x(k+i \mid k)
\end{aligned}
$$

for any $[A(k+i), B(k+i)] \in \Omega$.

Lemma 4 (see [4]). For system (1), if it has a minimum delay rate of (10), the following LMI must be satisfied:

$$
\left[\begin{array}{cc}
\rho^{2} Q & * \\
A_{i} Q+B_{i} Y & Q
\end{array}\right] \geq 0, \quad i=1, \ldots, L .
$$

Algorithm 5 (automatic offline robust MPC). Consider the uncertain system (1) subject to input and output constraints (2). A sequence of minimizers $\gamma_{i}, Q_{i}, X_{i}, Y_{i}$, and $Z_{i}$ are generated based on the sufficient large feasible initial state $x_{1}$ offline as follows:

Set $i:=1$.

(1) Compute the minimizer $\gamma_{i}, Q_{i}, X_{i}, Y_{i}, Z_{i}$ at $x_{i}$ by (9), with additional LMIs (11) and $\rho^{2} Q_{i}<Q_{i+1}<Q_{i}$, store $F_{i}$.

(2) If $i<N$, choose a state $x_{i+1}$ satisfying $x_{i+1}^{T} Q_{i}^{-1} x_{i+1}=$ $\rho^{2} x_{i}^{T} Q_{i}^{-1} x_{i}$. Let $i:=i+1$; go to step 1 .

Suppose the given initial state $x_{0}$ satisfying $\left\|x_{j+1}\right\|_{Q_{j}^{-1}} \leq$ $\left\|x_{0}\right\|_{Q_{j}^{-1}} \leq\left\|x_{j}\right\|_{Q_{j}^{-1}}, j \in\{1, \ldots, N\}$. Let the state be $x(k)$ at time $k$, and apply the control law

$$
u(k)= \begin{cases}F_{k+j} x(k) & k+j \leq N \\ F_{N} x(k) & k+j>N\end{cases}
$$

online.

Remark 6. Algorithm 5 is independent of the state. The control law is not optimal as it can be applied to all the states within the ellipsoid. The information of the exact smallest ellipsoid containing the current state is not needed before the application of the controller compared with the method in paper [8].

Lemma 7. Suppose the positive matrices satisfy the following condition: $0<\rho^{2} Q_{i}<Q_{i+1}<Q_{i}(0<\rho<1)$; then $\mathscr{E}_{i, \rho} \subset$ $\mathscr{E}_{i+1} \subset \mathscr{E}_{i}$, where $\mathscr{E}_{i}=\left\{x \in R^{n} \mid x^{T} Q_{i}^{-1} x \leq 1\right\}$ and $\mathscr{E}_{i, \rho}=$ $\left\{x \in R^{n} \mid x^{T} Q_{i}^{-1} x \leq \rho^{2}\right\}$.

Proof. If $x \in \mathscr{E}_{i, \rho}$, then $x^{T} Q_{i}^{-1} x<\rho^{2}$. As $0<\rho^{2} Q_{i}<Q_{i+1}$, it follows $\rho^{2} Q_{i+1}^{-1}<Q_{i}^{-1}$ and $\rho^{2} x^{T} Q_{i+1}^{-1} x<x^{T} Q_{i}^{-1} x<\rho^{2}$. Hence $x^{T} Q_{i+1}^{-1} x<1$ so that $x \in \mathscr{E}_{i+1}$ and therefore $\mathscr{E}_{i, \rho} \subset \mathscr{E}_{i+1}$;

If $x \in \mathscr{E}_{i+1}$, then $x^{T} Q_{i+1}^{-1} x<1$. Because $0<Q_{i+1}<Q_{i}$, it follows $Q_{i}^{-1}<Q_{i+1}^{-1}$. Hence $x^{T} Q_{i}^{-1} x<x^{T} Q_{i+1}^{-1} x<1$ so that $x \in \mathscr{E}_{i}$ and therefore $\mathscr{E}_{i+1} \subset \mathscr{E}_{i}$.

From above, it is easy to get $\mathscr{E}_{i, \rho} \subset \mathscr{E}_{i+1} \subset \mathscr{E}_{i}$. 


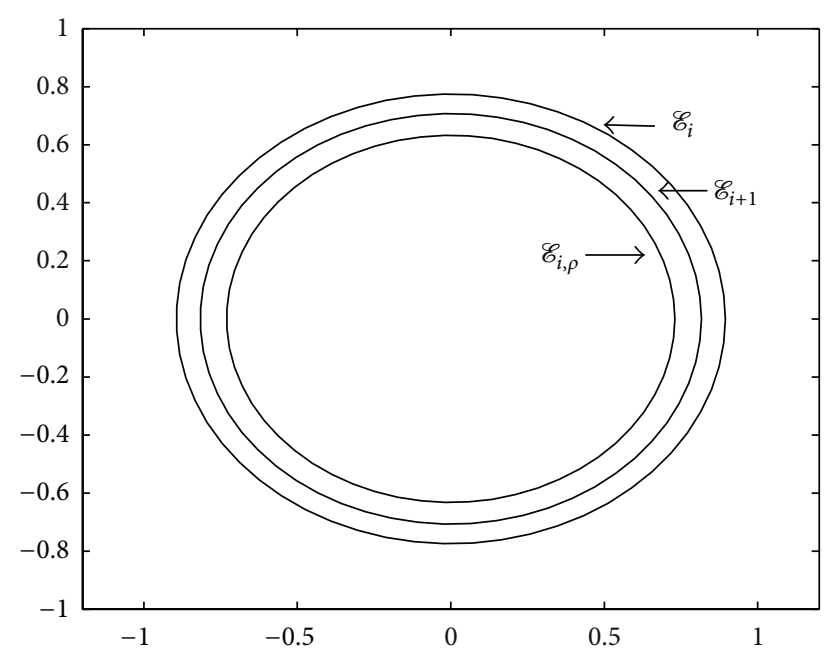

FIGURE 1: Relationship between $\mathscr{E}_{i}, \mathscr{E}_{i, \rho}$, and $\mathscr{E}_{i+1}$.

Theorem 8. Given a dynamical system (1) and a feasible initial state $x_{0}$ satisfying $\left\|x_{j+1}\right\|_{Q_{j}^{-1}} \leq\left\|x_{0}\right\|_{Q_{j}^{-1}} \leq\left\|x_{j}\right\|_{Q_{j}^{-1}}, j \in$ $\{1, \ldots, N\}$, the automatic offline robust MPC Algorithm 5 robustly asymptotically stabilizes the closed-loop system.

Proof. For the offline minimization at $x_{i}, i=1,2, \ldots, N$, the LMI (11) ensures that its convergence speed is not less than $\rho$. So, if $x(k) \in \mathscr{E}_{i}=\left\{x \in R^{n} \mid x^{T} Q_{i}^{-1} x \leq 1\right\}$, then $x(k+1) \epsilon$ $\mathscr{E}_{i, \rho}=\left\{x \in R^{n} \mid x^{T} Q_{i}^{-1} x \leq \rho^{2}\right\}$. From Lemma 7, the control law corresponding to the ellipsoid $\mathscr{E}_{i}$ is guaranteed to keep the state within $\mathscr{E}_{i}$ and converge into the ellipsoid $\mathscr{E}_{i+1}$, and so on. Lastly, $u_{N}=F_{N} x(k)$ is guaranteed to keep the state within $\mathscr{E}_{N}$ and converge to the origin.

Compared with the strategy in paper [8], the proposed algorithm reduces the conservatism but may sacrifice some optimality. For example, according to Algorithm 5, the state is in a smaller ellipsoid, but the feedback control law may be computed based on a larger ellipsoid, which is showed in Figure 1. So we propose a modified algorithm.

Algorithm 9 (modified automatic offline robust MPC). Consider the same system as Algorithm 5. Given a sufficiently large feasible state $x_{1}$, generate a sequence of minimizers $\gamma_{i}, Q_{i}, X_{i}, Y_{i}$, and $Z_{i}$ as follows offline. Let $i:=1$.

(1) Compute the minimizer $\gamma_{i}, Q_{i}, X_{i}, Y_{i}, Z_{i}$ at $x_{i}$ by solving the problem (9) with additional LMIs (11) and $\rho^{2} Q_{i}<Q_{i+1}<Q_{i}$, store $F_{i}$. If $i=[N / 2]$; store $Q_{i}^{-1}$.

(2) If $i<N$, choose a state $x_{i+1}$ satisfying $x_{i+1}^{T} Q_{i}^{-1} x_{i+1}=$ $\rho^{2} x_{i}^{T} Q_{i}^{-1} x_{i}$. Let $i:=i+1$; go to step 1 .

The modified control strategy is selected online. Suppose the initial state $x_{0}$ satisfying $\left\|x_{j+1}\right\|_{Q_{j}^{-1}}^{2} \leq\left\|x_{0}\right\|_{Q_{j}^{-1}}^{2} \leq$ $\left\|x_{j}\right\|_{\mathrm{Q}_{j}^{-1}}^{2}, j \in\{1, \ldots, N\}$. Let the state be $x(k)$ at time $k$.

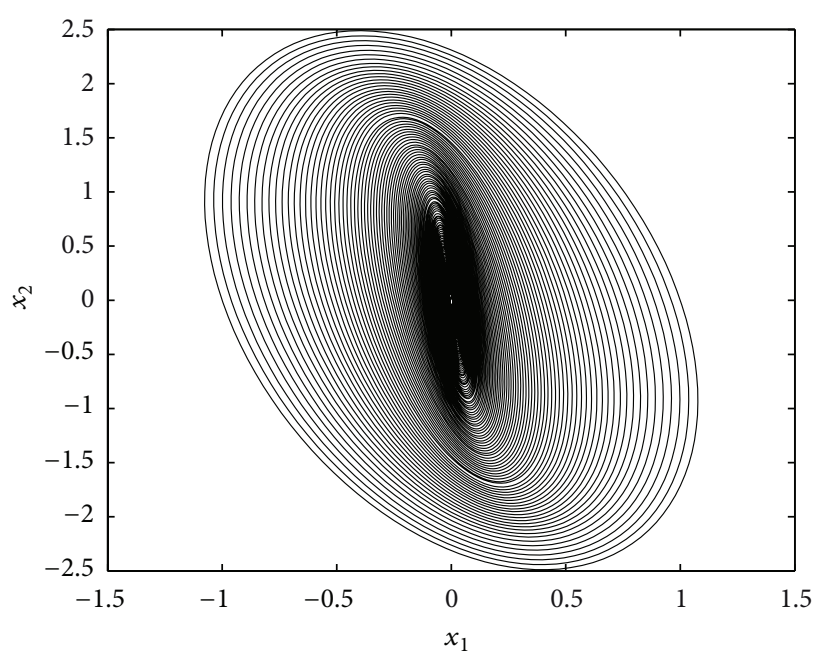

FIGURE 2: The ellipsoids defined by $Q_{i}^{-1}$ for all 150 discrete points for Example 12.

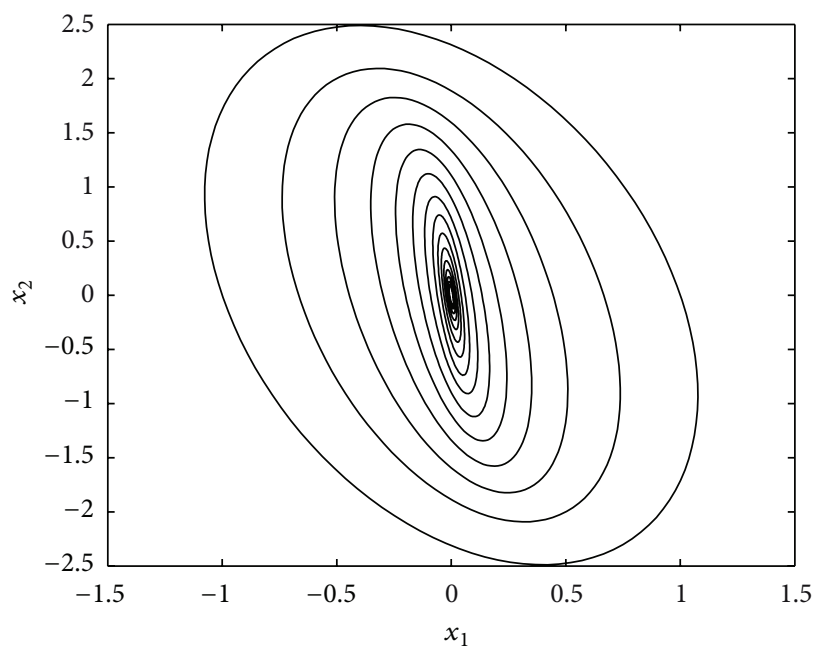

FIGURE 3: The ellipsoids defined by $Q_{i}^{-1}$ for every other ten ellipsoids discrete points for Example 12.

TABLE 1: The corresponding average computation time.

\begin{tabular}{lc}
\hline Algorithm & On-line average computation time \\
\hline TMPC & 2.84 \\
OLMPC & 0.011 \\
AOLMPC & 0.004 \\
\hline
\end{tabular}

Case 1. If $j \geq N / 2$, apply the control law

$$
u(k)= \begin{cases}F_{k+j} x(k) & k+j \leq N \\ F_{N} x(k) & k+j>N .\end{cases}
$$

Case 2. If $j<N / 2$,

(1) select $u(k)=F_{k+j} x(k)$;

(2) compute $x(k+1)^{T} Q_{[N / 2]}^{-1} x(k+1)$; if $x(k+1)^{T} Q_{[N / 2]}^{-1} x(k+1)>1, k:=k+1$; go to step 1 ; 


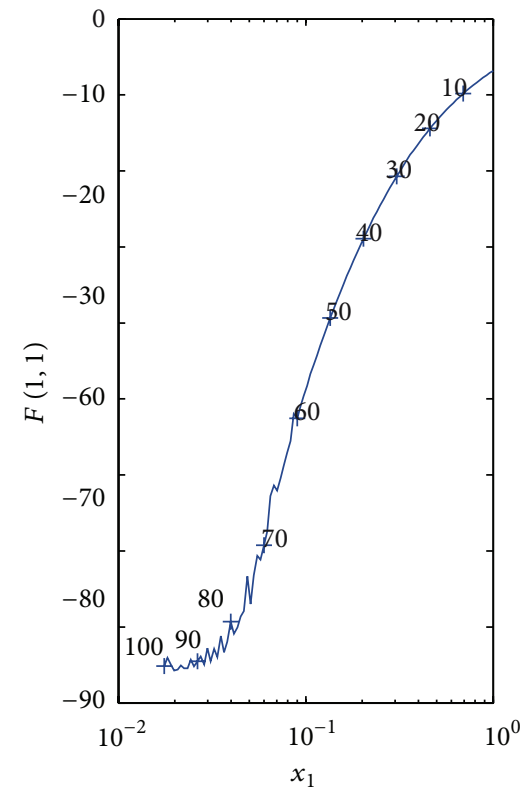

(a)

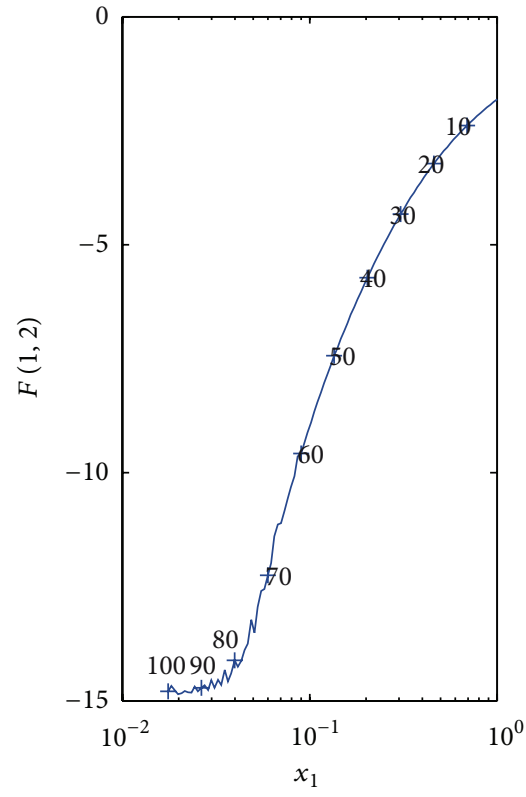

(b)

Figure 4: The offline control law $F$ for Example 12.

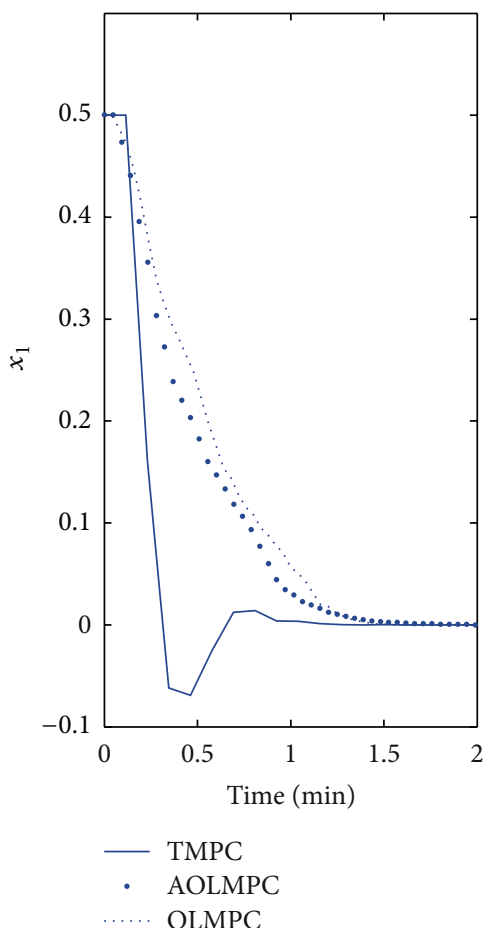

(a)

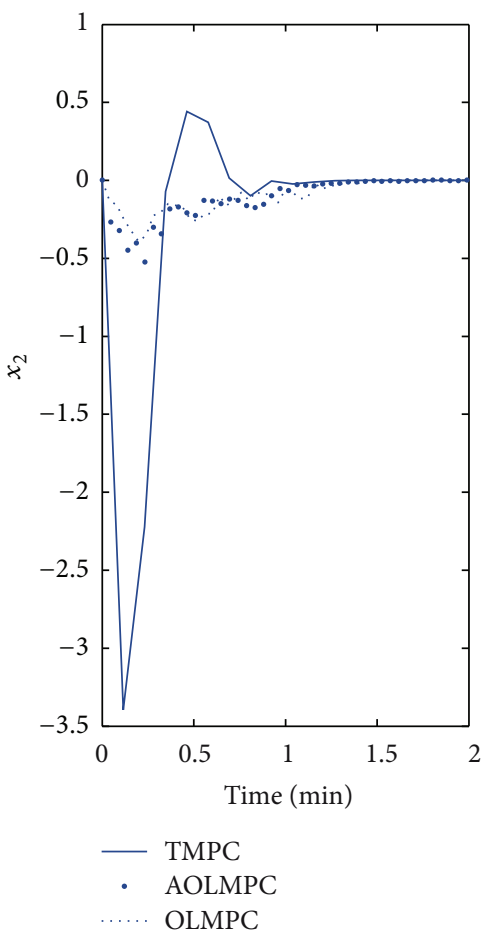

(b)

FIGURE 5: Closed-loop responses for Example 12.

TABLE 2: The convergence speed.

\begin{tabular}{lcccc}
\hline$k$ & 1 & 10 & 20 & 30 \\
\hline$x(k)$ & {$[0.500 ; 0]$} & {$[0.2254 ;-0.1997]$} & {$[0.0651 ;-0.1038]$} & {$[-0.0004 ;-0.0054]$} \\
$\Phi(k)=x(k)^{T} Q_{k}^{-1} x(k)$ & 0.2499 & 0.0876 & 0.0152 & $2.3162 e-005$ \\
$\Phi(k+1) / \Phi(k)$ & 0.3504 & 0.1964 & 0.0019 & 0.0306 \\
\hline
\end{tabular}


if $x(k+1)^{T} Q_{[N / 2]}^{-1} x(k+1) \leq 1$, select the following controller $u(k)=F_{[N / 2]} x(k), k^{\prime}:=k$; go to step 3 ;

(3) $k:=k+1$, select

$$
u(k)= \begin{cases}F_{[N / 2]+k-k^{\prime}} x(k) & {\left[\frac{N}{2}\right]+k-k^{\prime} \leq N} \\ F_{N}(k) & {\left[\frac{N}{2}\right]+k-k^{\prime}>N .}\end{cases}
$$

Remark 10. Algorithm 9 enforces the optimality an as it sets observation point. It ought to set more points to get more points to get the better performance. But the added points may increase the online computation and need more information of the system. The number of the observation points depends on the real system. How to select the proper number of observation points depends on the control engineering practice.

Remark 11. The online execution time for Algorithm 5 does not depend on the size of the system, so it has constant time (also written as $O(1)$ time). For the method in paper [8], the total number of flops required to calculate an input move is $\mathrm{O}\left(n_{s}^{2} \log _{2} K\right)$ for a sequence of $K$, with $n_{s}$ being the number of state variables. So we can conclude that this offline approach can dramatically reduce the online computational burden.

\section{Numerical Example}

In this section, two examples are presented to illustrate the effectiveness of the automatic offline approach. The simulations were performed on a Sony PC with an Intel Core i5 3317U processor $(1.7 \mathrm{GHz}$, memory $4 \mathrm{~GB})$ and using the software LMI control Toolbox in the MATLAB 7.0 environment to compute the solution of the linear minimization problem.

Example 12. Consider the system in [8] represented as

$$
\begin{aligned}
x(k+1) & =\left[\begin{array}{c}
x_{1}(k+1) \\
x_{2}(k+1)
\end{array}\right] \\
& =\left[\begin{array}{cc}
1 & 0.1 \\
0 & 1-0.1 \alpha(k)
\end{array}\right] x(k)+\left[\begin{array}{c}
0 \\
0.1 \kappa
\end{array}\right] u(k) \\
& \triangleq A(k) x(k)+B(k) u(k) \\
y(k) & =\left[\begin{array}{ll}
1 & 0
\end{array}\right] x(k) \triangleq C x(k)
\end{aligned}
$$

where $\kappa=0.787,0.1 \leq \alpha \leq 10, A(k) \in \Omega=\operatorname{Co}\left\{A_{1}, A_{2}\right\}$, $A_{1}=\left[\begin{array}{ll}1 & 0.1 \\ 0 & 0.99\end{array}\right]$, and $A_{2}=\left[\begin{array}{cc}1 & 0.1 \\ 0 & 0\end{array}\right]$.

The robust performance objective function (3) is subject to $|u(k+i \mid k)| \leq 2, i \geq 0$. Here $J_{\infty}(k)$ is given by (4) with

$$
Đ=\left[\begin{array}{ll}
1 & 0 \\
0 & 0
\end{array}\right], \quad R=0.00002 .
$$

We choose the $x_{1}$-axis as the one-dimensional subspace and discretize it according to the fixed geometric proportion, for example, with $\rho=0.96$. That is $x_{1}^{\text {set }}=\left\{z_{i}\right\}$, with $z_{1}=1, z_{i+1}=$

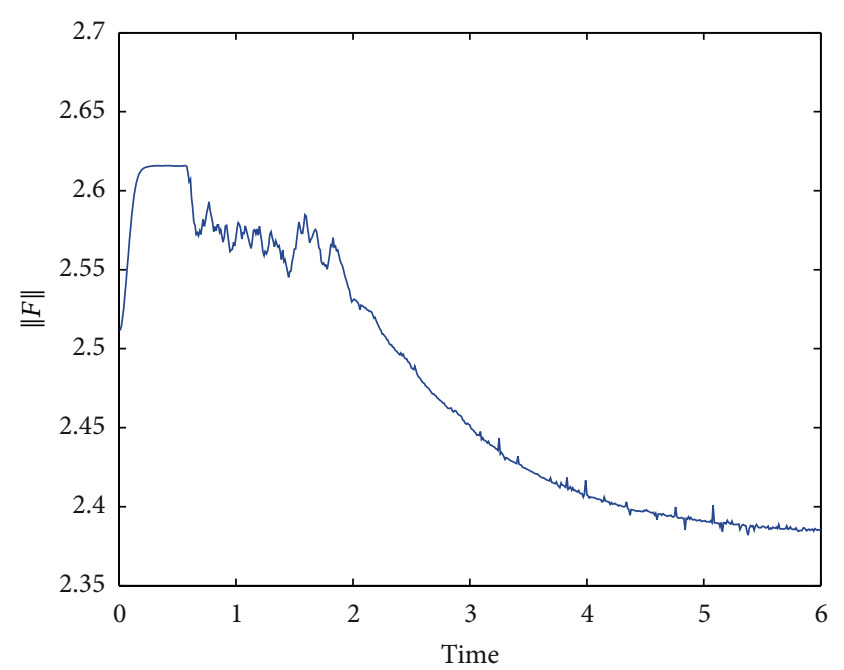

FIgURE 6: Feedback matrix norm for Example 13.

$\rho z_{i}, i=1,2,3, \ldots$. Figure 2 shows the ellipsoids defined by $Q_{i}^{-1}$ for all 150 discrete points. As it is too dense, an ellipsoid every other ten ellipsoids is selected and it is illustrated in Figure 3.

Figure 4 shows the computed offline control law along the $x_{1}$-axis. Both plots are obtained using the offline part of Algorithm 5. As the step $i$ increases, the feedback almost keeps constant $(i>90)$. This is corresponding to the system's unconstrained control.

Given the initial state $x(0)=\left[\begin{array}{ll}0.5 & 0\end{array}\right]^{T}$, Figure 5 shows the closed-loop responses of the system under the traditional model predictive control [4] (TMPC), offline model predictive control (OLMPC) [8], and automatic offline model predictive control (AOMPC) respectively. It shows that the AOLMPC, as it is based on the geometric proportion asymptotically stable ellipsoid, has the less overshoot. The average online computation time of the proposed algorithm compared with the other two methods is listed in Table 1.

The system's convergence speed at step $k=1,10,20,30$ is shown in Table 2. As $0.96^{10}=0.6648$, it is concluded that the average convergence rate is more than 0.96 from Table 2.

Example 13. Consider the two-mass-spring benchmark problem [19] represented as

$$
\begin{gathered}
{\left[\begin{array}{c}
\dot{x}_{1} \\
\dot{x}_{2} \\
\dot{x}_{3} \\
\dot{x}_{4}
\end{array}\right]=\left[\begin{array}{cccc}
0 & 0 & 1 & 0 \\
0 & 0 & 0 & 1 \\
\frac{-k}{m_{1}} & \frac{k}{m_{1}} & 0 & 0 \\
\frac{k}{m_{2}} & \frac{-k}{m_{2}} & 0 & 0
\end{array}\right]\left[\begin{array}{l}
x_{1} \\
x_{2} \\
x_{3} \\
x_{4}
\end{array}\right]+\left[\begin{array}{c}
0 \\
0 \\
\frac{1}{m_{1}} \\
0
\end{array}\right] u} \\
y=x_{2},
\end{gathered}
$$

where $m_{1}=m_{2}=1,0.5 \leq k \leq 1.2$, and $|u| \leq 1$. Using Algorithm 5 and choosing the initial state $x(0)=$ $\left[\begin{array}{llll}-1 & -1 & 0 & 0\end{array}\right]^{T}, Ð=\operatorname{diag}(0,1,0,0), R=0.1$, we can get the GPASE using Algorithm 5. The norm of the feedback matrix and the states are exhibited in Figures 6 and 7, respectively. 


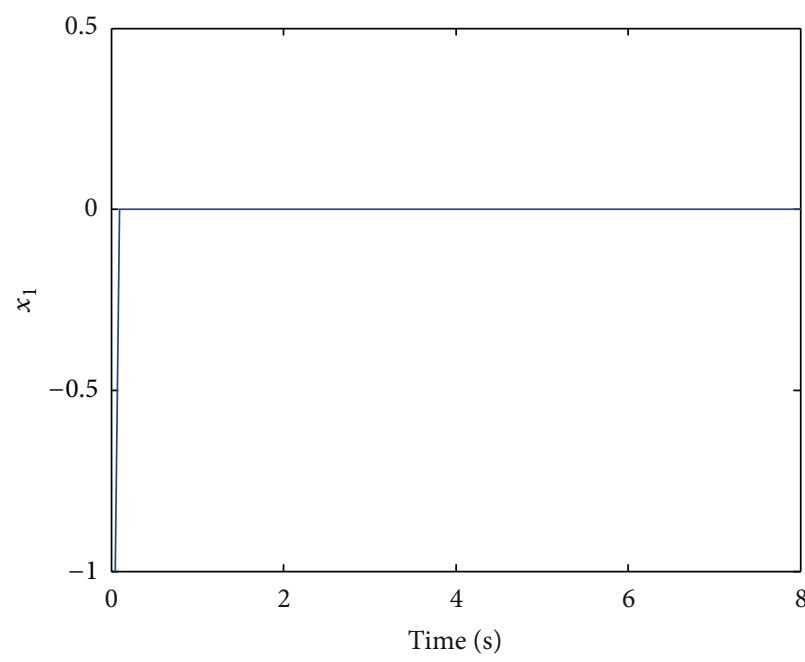

(a)

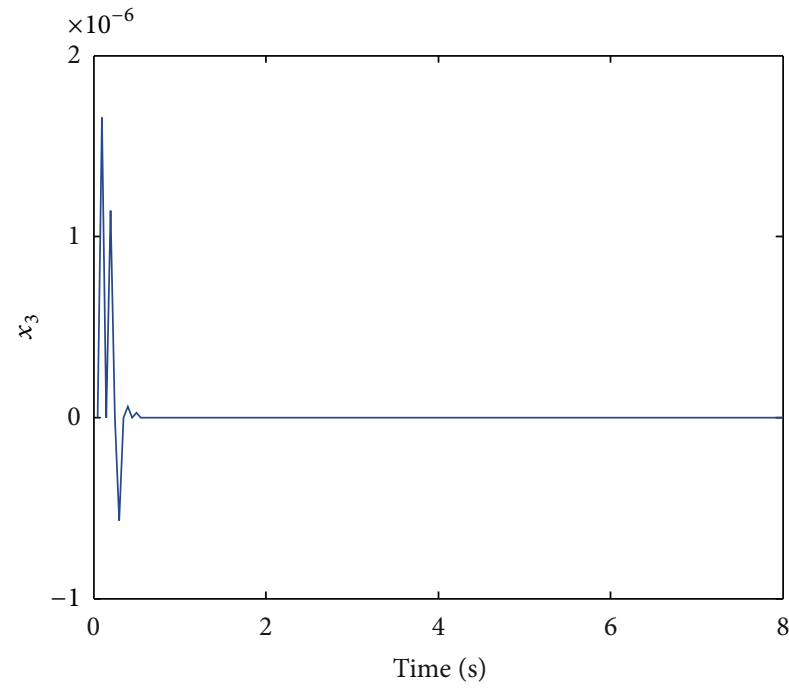

(c)

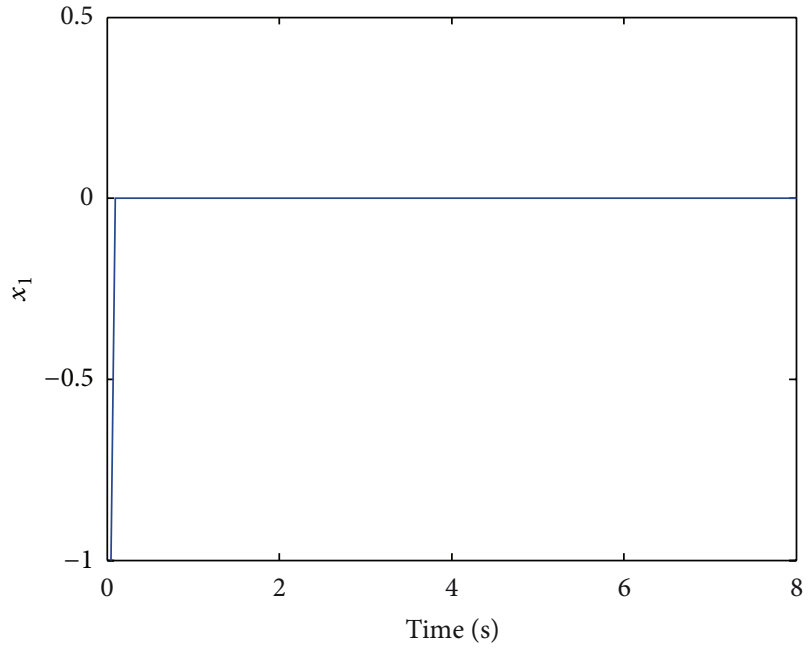

(b)

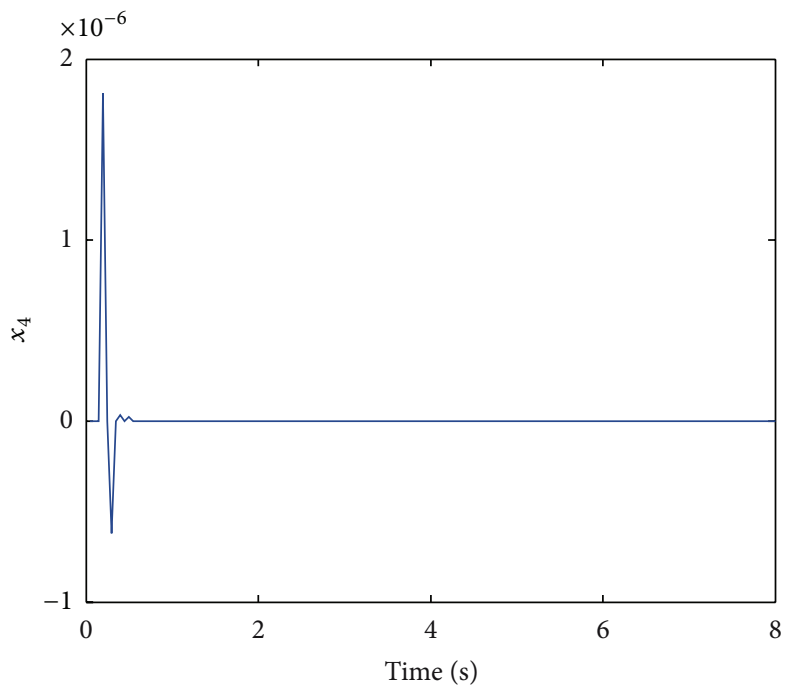

(d)

FIgURE 7: State trajectory for Example 13.

\section{Conclusions}

An automatic stable closed-loop robust MPC strategy for uncertainty system with input and output constraints has been developed. This method not only reduces the conservativeness dramatically, but also reduces the online computation. The stability of this technique is proved based on GPASE. Two examples indicate the effectiveness of this method.

\section{Acknowledgments}

This work was supported by the National Natural Science Foundation of China under Grant 60974051, 61273144, Natural Science Foundation of Beijing under Grant 4122071, Fundamental Research Funds for the Central Universities under Grant 12MS143, and the Construction Project from Beijing Municipal Commission of Education.

\section{References}

[1] S. J. Qin and T. A. Badgwell, "A survey of industrial model predictive control technology," Control Engineering Practice, vol. 11, no. 7, pp. 733-764, 2003.

[2] D. Q. Mayne, J. B. Rawlings, C. V. Rao, and P. O. M. Scokaert, "Constrained model predictive control: stability and optimality," Automatica, vol. 36, no. 6, pp. 789-814, 2000.

[3] A. Bemporad and M. Morari, "Robust model predictive control: a survey," in Robustness in Identification and Control, A. Garulli, A. Tesi, and A. Vicino, Eds., vol. 245, pp. 207-226, Springer, London, UK, 1999.

[4] M. V. Kothare, V. Balakrishnan, and M. Morari, "Robust constrained model predictive control using linear matrix inequalities," Automatica, vol. 32, no. 10, pp. 1361-1379, 1996.

[5] A. Bemporad, F. Borrelli, and M. Morari, "Min-max control of constrained uncertain discrete-time linear systems," Institute of Electrical and Electronics Engineers, vol. 48, no. 9, pp. 1600-1606, 2003. 
[6] V. Sakizlis, N. M. P. Kakalis, V. Dua, J. D. Perkins, and E. N. Pistikopoulos, "Design of robust model-based controllers via parametric programming," Automatica, vol. 40, no. 2, pp. 189201, 2004

[7] D. Muñoz de la Peña, A. Bemporad, and C. Filippi, "Robust explicit MPC based on approximate multiparametric convex programming," Institute of Electrical and Electronics Engineers, vol. 51, no. 8, pp. 1399-1403, 2006.

[8] Z. Wan and M. V. Kothare, "An efficient off-line formulation of robust model predictive control using linear matrix inequalities," Automatica, vol. 39, no. 5, pp. 837-846, 2003.

[9] B. Ding, Y. Xi, M. T. Cychowski, and T. O’Mahony, "Improving off-line approach to robust MPC based-on nominal performance cost," Automatica, vol. 43, no. 1, pp. 158-163, 2007.

[10] D. Angeli, A. Casavola, G. Franzè, and E. Mosca, "An ellipsoidal off-line MPC scheme for uncertain polytopic discrete-time systems," Automatica, vol. 44, no. 12, pp. 3113-3119, 2008.

[11] B. Kouvaritakis, J. A. Rossiter, and J. Schuurmans, "Efficient robust predictive control," Institute of Electrical and Electronics Engineers, vol. 45, no. 8, pp. 1545-1549, 2000.

[12] X. Liu, S. Feng, and M. Ma, "Robust MPC for the constrained system with polytopic uncertainty," International Journal of Systems Science, vol. 43, no. 2, pp. 248-258, 2012.

[13] J. Schuurmans and J. A. Rossiter, "Robust predictive control using tight sets of predict -ted states, control theory and applications," IEE Proceedings, vol. 147, no. 1, pp. 13-18, 2000.

[14] L. Zhang, X. Liu, and X. Kong, "State estimators for uncertain linear systems with different disturbance/noise using quadratic boundedness," Journal of Applied Mathematics, vol. 2012, Article ID 101353, 10 pages, 2012.

[15] Z. Y. Wan and M. V. Kothare, "Robust output feedback model predictive control using off-line linear matrix inequalities," Journal of Process Control, vol. 12, no. 7, pp. 763-774, 2002.

[16] M. A. Rodrigues and D. Odloak, "Robust MPC for systems with output feedback and input saturation," Journal of Process Control, vol. 15, no. 7, pp. 837-846, 2005.

[17] B. Ding, Y. Xi, M. T. Cychowski, and T. O’Mahony, "A synthesis approach for output feedback robust constrained model predictive control," Automatica, vol. 44, no. 1, pp. 258-264, 2008.

[18] B. Ding, "Constrained robust model predictive control via parameter-dependent dynamic output feedback," Automatica, vol. 46, no. 9, pp. 1517-1523, 2010.

[19] Z. Wan and M. V. Kothare, "Efficient robust constrained model predictive control with a time varying terminal constraint set," Systems \& Control Letters, vol. 48, no. 5, pp. 375-383, 2003. 


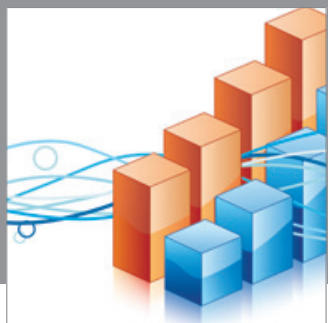

Advances in

Operations Research

mansans

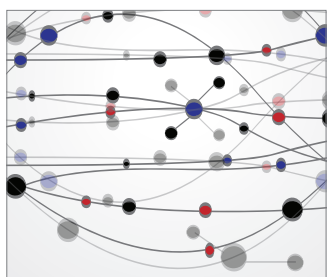

The Scientific World Journal
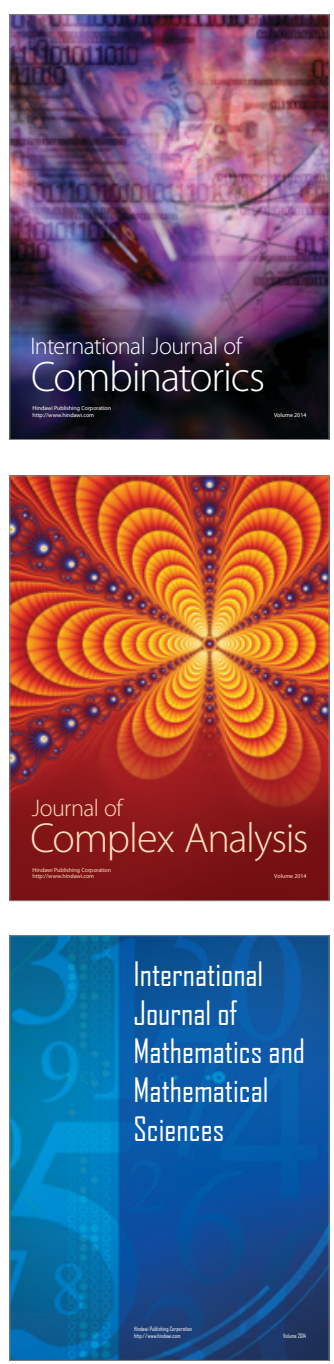
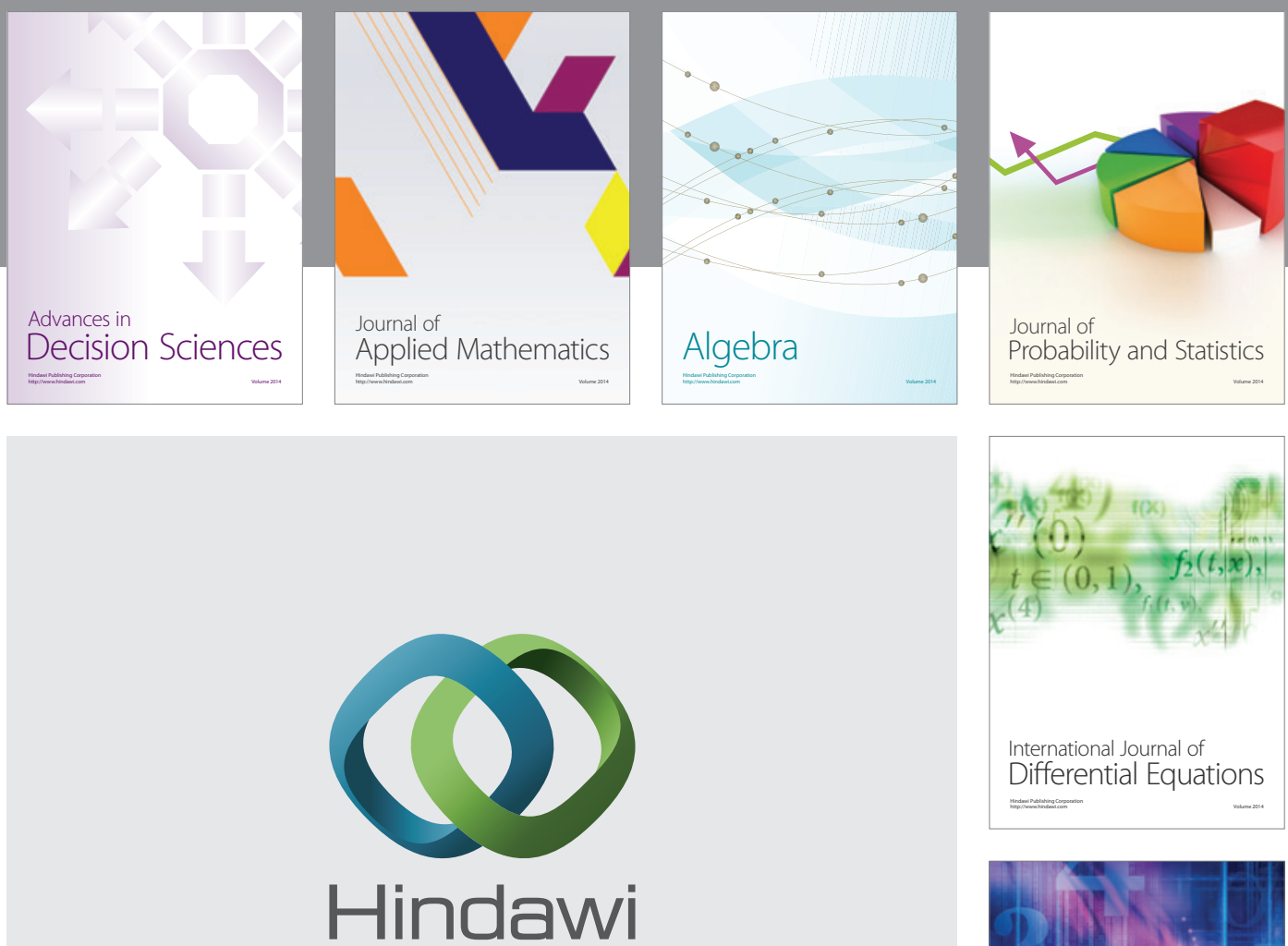

Submit your manuscripts at http://www.hindawi.com
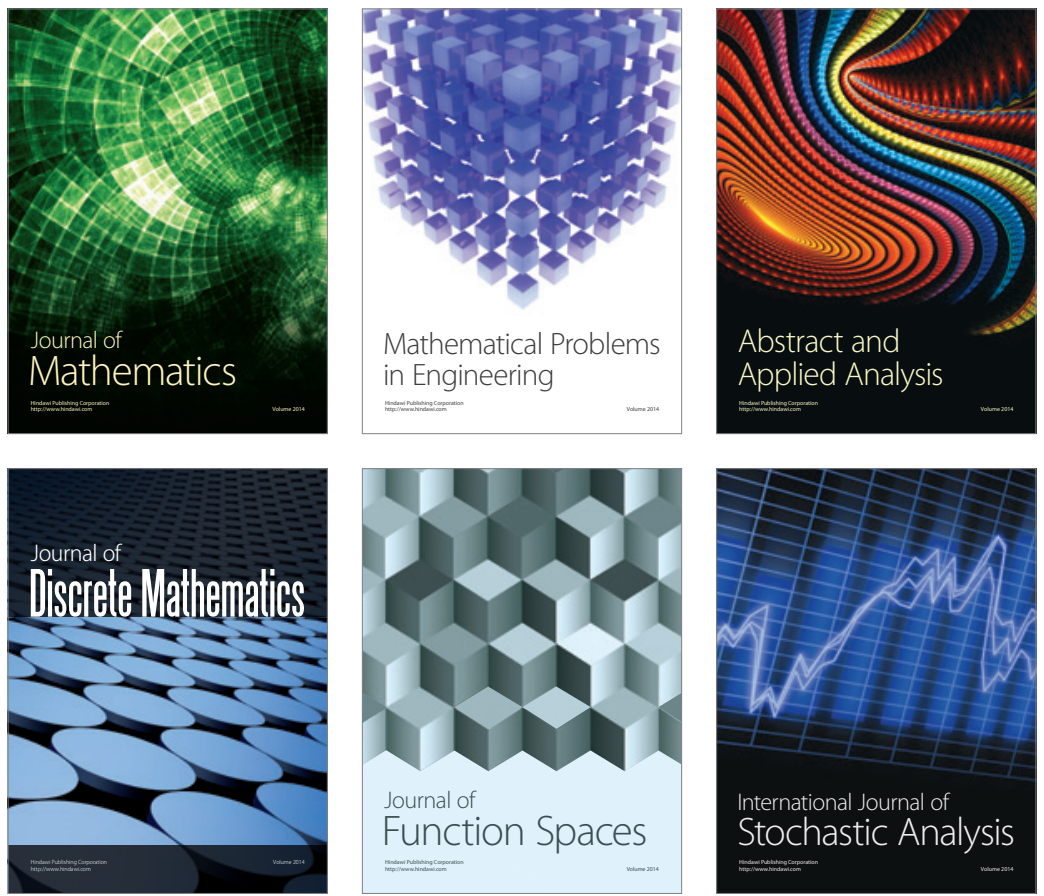

Journal of

Function Spaces

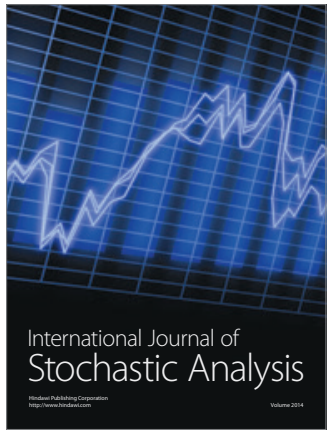

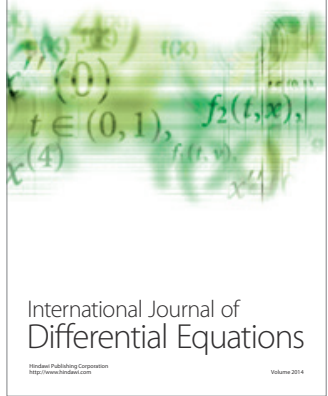
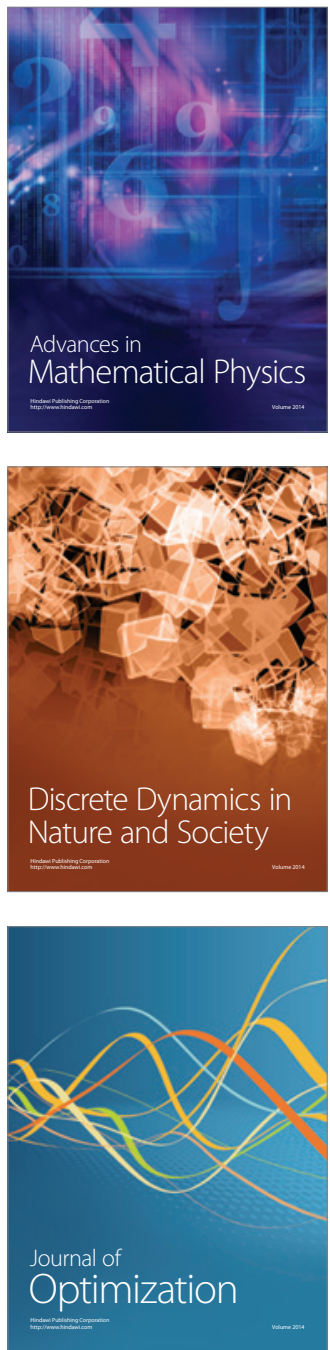